\title{
Distinct copulation positions in Drosophila pachea males with symmetric or asymmetric external genitalia
}

\author{
Michael Lang ${ }^{1}$, Virginie Orgogozo ${ }^{1,2}$ \\ ${ }^{1}$ CNRS UMR7592, Université Paris Diderot, Institut Jacques Monod, Bâtiment Buffon 444B, 15 rue Hélène Brion, \\ 75205 Paris cedex 13, France \\ ${ }^{2}$ E-mail: orgogozo@ijm.univ-paris-diderot.fr
}

Key words: epandrial lobe, epandrium, left-right asymmetry, mating behaviour, mating position, nannoptera subgroup

\begin{abstract}
Left-right asymmetric genitalia have appeared multiple times independently in insects and have been associated with changes in mating positions. However, there is little experimental data on how the evolution of genital asymmetries may have affected the evolution of mating positions or vice versa. As opposed to its closely-related species, Drosophila pachea has a conspicuous asymmetry in its male genitalia external lobes, with the left lobe being $1.49 \pm 0.08$ (SD) times longer and thinner than the right lobe. In a laboratory stock, we found that $20 \%$ of the males possess fully symmetric lobes. To better understand how asymmetric genitalia may affect mating, we compared $D$. pachea copulation behaviour between these mutant males and wildtype males. We found that $D$. pachea wild-type males adopt a one-sided mating posture with the male always one-sided $8.55^{\circ}$ $\pm 1.79^{\circ}$ (SD) towards the female's right side. Within 45 -min recordings, all wild-type males did mate whereas $39 \%$ of symmetric mutants failed to form a stable mating complex and did not mate. In successful copulations, symmetric mutants also adopted a right-sided mating posture but the angle between male and female bodies was significantly more variable compared to wild-type males. Our results suggest that lobe size asymmetry is required for the formation of a stable mating complex and for the positioning of the male according to a precise angle on the female. However, lobe size asymmetry is not required for $D$. pachea right-sided mating posture.
\end{abstract}

\section{Contents}

Introduction 87

Material and methods ................................................................ 89

Fly stock and adult maintenance ...................................... 89

Crosses and copulation monitoring ................................. 89

Genitalia preparation and microscopy ........................... 90

Results

The D. pachea male epandrium is asymmetric 91

The D. pachea copulation posture is asymmetric .......... 91

One D. pachea stock contains mutant males

with symmetric lobes

The copulation position of males

with symmetric genitalia is atypical and unsteady ........ 91

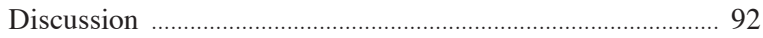

Drosophila pachea males mate

on the female's right side ...................................................... 92

Lobe function in mating ....................................................... 92

Drosophila pachea males with symmetric genitalia

still mate on the female's right side ................................. 93

Evolution of mating positions ............................................. 93

Acknowledgements ............................................................... 94

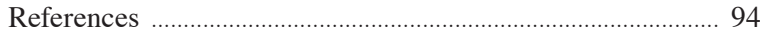

\section{Introduction}

Left-right asymmetries have been identified in phylogenetically diverse insect species and likely arose multiple times independently (Alexander, 1964; McAlpine, 1981; Huber et al., 2007). Whereas in most dipteran species the body is entirely symmetric, a few species exhibit an asymmetry in organ size, which is almost always restricted to the male or female adult terminalia (McAlpine, 1981). Various explanations have been proposed for the evolution of asymmetric genitalia in insects, including space constraints, ecological constraints and arms race between males and females, but the most favoured one is sexual selection via changes in mating position (Alexander, 1964; McAlpine, 1981; Huber et al., 2007; Huber, 2010). When primitive arthropods started to colonize land, fertilization is thought to have evolved from external fertilization in water to the use of spermatophores that were deposited on the soil by males and taken up by females. Then, copulation probably evolved from spermatophore transferral. An amazing diversity of mating positions is found today across insects and in Diptera in particular (Alexander, 1964). Based mainly on variations in mating positions across different insect taxa and on changes in position during mating, it was suggested that the ancestral Neopteran copulation position was with both 
male and female facing in the same direction and the female on top. Derived mating positions then likely evolved as a result of selection for males to have more control over the female, leading to male-on-side or -on-top positions (Alexander, 1964; McAlpine, 1981; Huber, 2010). Such an ancestral female-on-top configuration is compatible with the ventral location of most female genital openings and with copulation being evolutionarily derived from spermatophore transferral. As nicely explained by Huber (2010), derived evolutionary changes in mating positions probably required twisting of the male abdomen and asymmetric contacts of male and female genitalia during initial coupling. Likely consequences were compensatory morphological changes to reduce mismatch, functional diversification of left and right sides of genitalia, or even one-sided reduction (Huber, 2010). However, no formal cladistic analysis of mating position across the insect phylogeny has been reported, or at least within a taxon that includes species with the hypothesized ancestral female-up configuration. Given the rapid evolution of left-right asymmetries and mating positions, such a study is absolutely required to test the evolutionary scenario proposed by Alexander (1964), McAlpine (1981) and Huber (2010).

Additional support for this scenario comes from Cyclorrhaphan flies that have evolved a stereotyped mating posture, with both male and female facing towards the same direction and the male on top (Mc Alpine, 1981). Such a position coincides with a $360^{\circ}$ rotation of the Cyclorrhaphan male genitalia during metamorphosis (Brühl, 1897; Feuerborn, 1922; Schräder, 1927). This $360^{\circ}$ rotation is thought to have occurred sequentially during evolution, first with a $180^{\circ}$ rotation associated with a change mating position from female-above to tail-to-tail, and then with a second
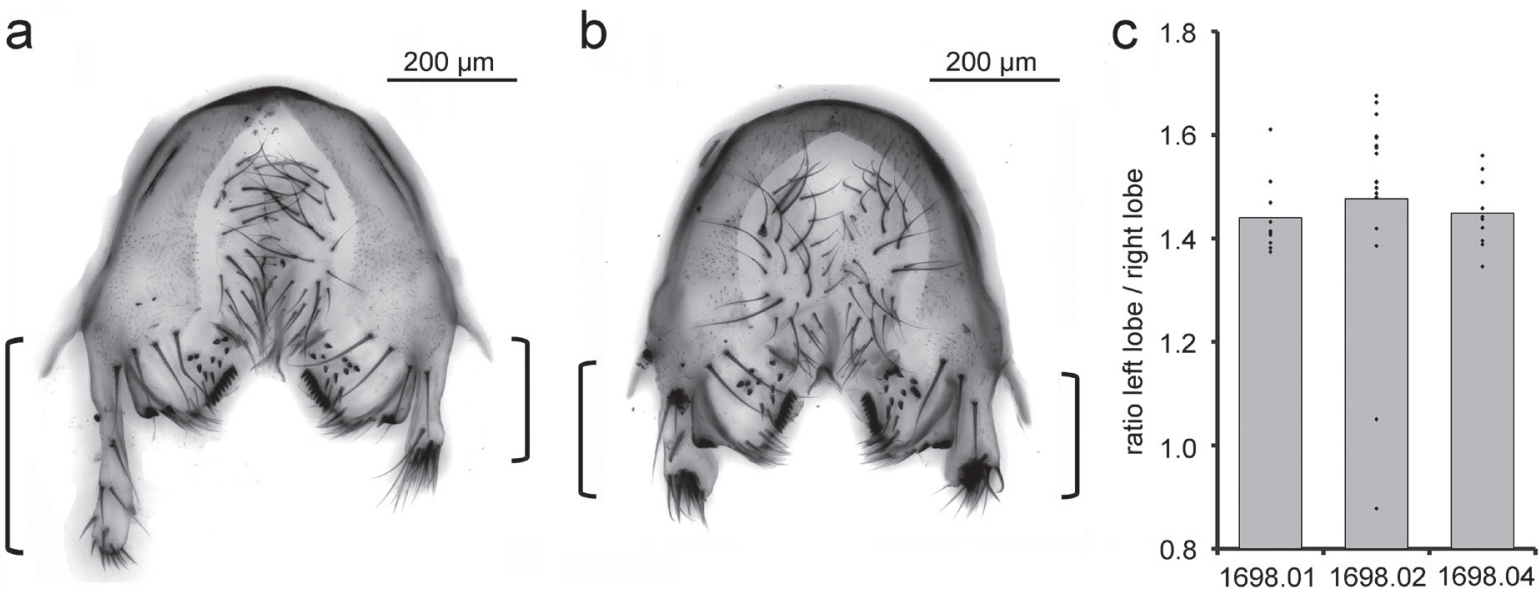

Fig. 1. Drosophila pachea epandrial lobes. Dissected epandrium of D. pachea wild-type (a) and symmetric mutant males (b) from stock 15090-1698.02. Left and right lobes are indicated by brackets. (c) Length ratios of left/right epandrial lobes of $D$. pachea from three laboratory stocks. Each dot corresponds to one individual.
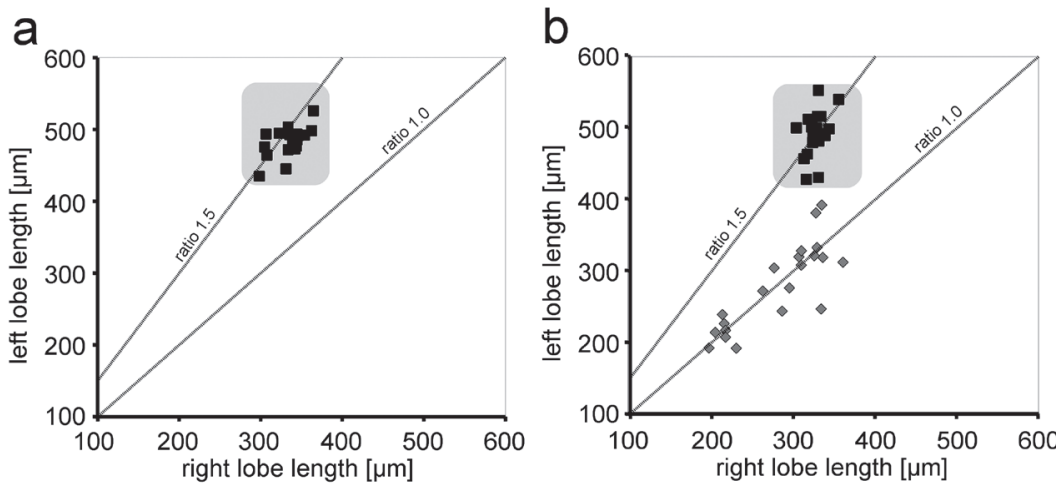

Fig. 2. Lobe length in wild-type and symmetric mutant males. Each individual is represented by a dot according to its left lobe and right lobe length. Ten individuals from stocks 15090-1698.01 and ten from stock 15090-1698.04 are shown in (a). Forty individuals from stock 150901698.02 are shown in (b). Those were used for copulation monitoring. Individuals were considered as mutants when they fell out of the range of lobe lengths observed in stocks 15090-1698.01 and 150901698.04 (grey area). 
$180^{\circ}$ rotation associated with a change from tail-to-tail position to the classic Cyclorrhaphan male-on-top position (Alexander, 1964; Huber, 2010; Suzanne et al., 2010). In all the proposed evolutionary scenarios, mating positions are thought to co-evolve with genital morphologies. However, it remains unknown and untested whether genital morphological changes have driven copulation posture changes or vice versa, or whether morphology and mating posture evolved simultaneously.

Experimental work with D. melanogaster (Meigen, 1830) showed that genital structures appear to function in proprioception and guidance of the couple in mating complex formation and mating position (Acebes et al., 2003). Ablation of the left or right large mechanosensory bristle on the male clasper led to adoption of an abnormal one-sided mating position. No other functional studies so far have evaluated how genital morphology, and asymmetry in particular, may affect copulation position.

In this study, we focused on Drosophila pachea (Patterson and Wheeler, 1942), which displays a striking left-right asymmetry in its male external genitalia (Pitnick and Heed, 1994). The male epandrium has a bilateral but asymmetric pair of external lobes, with the left lobe longer and thinner than the right one. This structure is specific to D.pachea and has likely evolved after the separation of this species from its sister species (Pitnick and Heed, 1994).

Drosophila pachea belongs to the nannoptera species group, which consists of four described cactusbreeding species: Drosophila nannoptera (Wheeler, 1949), D. acanthoptera (Wheeler, 1949), D. wassermanni (Pitnick and Heed, 1994) and D. pachea. These species are found in arid regions of west Mexico, but also in central and northern parts of South America. D. pachea is endemic to the Sonoran desert and the Baja California peninsula in north-west Mexico and is an obligate specialist to its plant host, the senita cacus Pachycereus schottii because of a physiologic dependence on 7-dehydrogenated sterols that are provided by the cactus (Heed and Kircher, 1965). Although asymmetric genitalia seem to be rare in Drosophilidae (Vilela and Baechli, 1990; Huber et $a l ., 2007)$, they are surprisingly common in the nannoptera species group. Whereas $D$. pachea displays an epandrial lobe size asymmetry, D. acanthoptera possesses an asymmetric phallus (Vilela and Baechli, 1990) and D. wassermanni a left concave- and a right convex-shaped cercus (Pitnick and Heed, 1994). Therefore, the nannoptera species group is an inter- esting system to study the evolution of asymmetric morphologies.

The mating positions in the nannoptera group species have not been described. Their bizarre genital structures motivated us to investigate $D$. pachea mating behaviour and to test for variation in the classic cyclorrhaphan mating position. We show that $D$. pachea has evolved a stereotyped right-sided male mating position. We also identified a new $D$. pachea mutant with males possessing symmetric lobes and used it to investigate the effect of genitalia asymmetry on copulation position. Our careful comparison of copulation positions between these mutant males and asymmetric wild-type males brings new insights into how genital asymmetry can affect copulation position.

\section{Material and methods}

\section{Fly stock and adult maintenance}

All Drosophila pachea stocks were retrieved from the San Diego Drosophila stock center (15090-1698.01, 15090-1698.02, 15090-1698.04) and were raised at $25^{\circ} \mathrm{C}$ in vials with $10 \mathrm{ml}$ of standard Drosophila medium, mixed with $150 \mu \mathrm{g}$ of 7-dehydrocholesterol (Sigma) dissolved in $30 \mu l$ of ethanol (Warren et al., 2001).

\section{Crosses and copulation monitoring}

Freshly emerged virgin adults from stock 150901698.02 were $\mathrm{CO}_{2}$-anaesthetized and separated according to gender and male genitalia. Males were left to mature for 14-20 days and females for 3-4 days (Pitnick et al., 1991). Each male was isolated into a single vial for at least two days prior to each mating recording.

Copulation recordings were performed in a selfmade climate controlled chamber at $25 \pm 0.5^{\circ} \mathrm{C}$, at 60 $\pm 5 \%$ humidity. Each male was tested for copulation once with a single virgin female in circular plastic cells (diameter: $10 \mathrm{~mm}$, height: $4 \mathrm{~mm}$ ) covered with transparent 1-mm plexiglas. Couples were observed from above with a Conrad 191251-62 digital camera for at least $45 \mathrm{~min}$ or until copulation ended. After copulation, adults were stored in absolute ethanol at $-20^{\circ} \mathrm{C}$. Images were exported manually from each recording every 2 minutes once the males started to mount the female. From 19 recordings of mating couples with wild-type males, we excluded 7 movies where flies were out of focus or positioned on the side 
of the plastic cell. From 23 recordings of mating couples with symmetric mutant males, we excluded 3 recordings for similar reasons and in 9 cases the flies did not form a mating complex (see results). The angle between female and male body midlines was drawn manually and quantified using the TpsDIG2. 16 software (F.J. Rohlf, http://life.bio.sunysb.edu/ morph/).

\section{Genitalia preparation and microscopy}

The distal part of male abdomen was cut from the adult and rehydrated for $30 \mathrm{~min}$ in distilled water. Male epandria were dissected with fine needles under a Zeiss Stemi 2000 stereomicroscope and mounted on a microscope slide in DMHF mounting medium (Entomopraxis A9001). Images of genital preparations
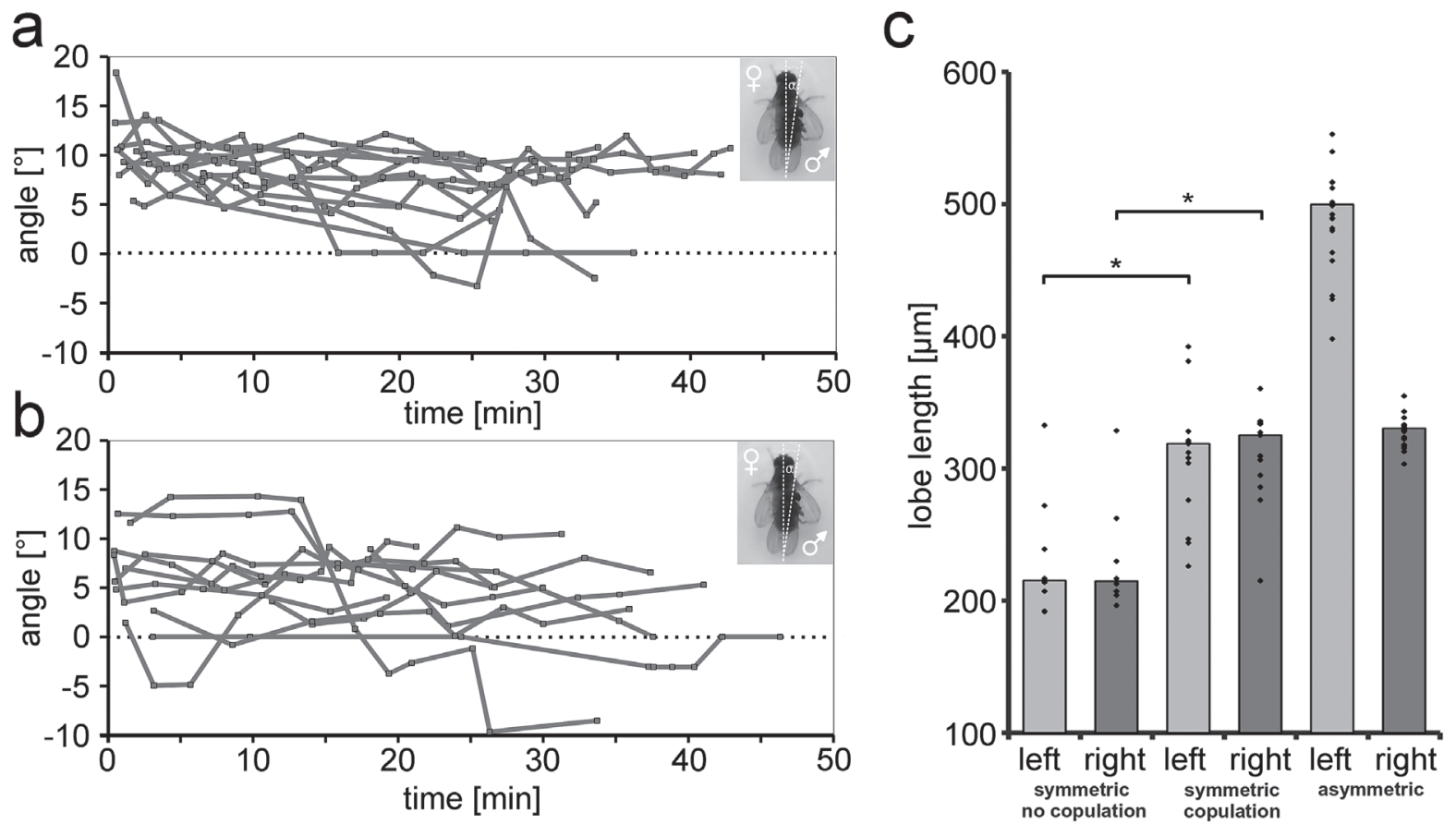

Fig. 3. Position of D. pachea males during copulation. Mating angles are presented over copulation time for couples with asymmetric wild-type males (a) and for couples with symmetric mutant males (b). The mating angle is defined as the angle between male and female midlines, see inset in (a) and (b). (c) Median left and right lobe lengths of recorded males that failed to copulate and of the ones that did copulate. $*=p<0.005$.

Table 1. Comparison of mating behaviour between wild-type and symmetric mutant males ( $\mathrm{SD}=$ standard deviation).

\begin{tabular}{lccc}
\hline Measurement & Asymmetric male & Symmetric male & F-Test \\
\hline $\begin{array}{l}\text { Number of analyzed couples } \\
\text { Number of males which failed to copulate }\end{array}$ & 19 & 22 \\
$\begin{array}{l}\text { Average number of unsuccessful copulation attempts } \\
\quad \text { before successful mating }\end{array}$ & 0 & 8 & \\
$\begin{array}{l}\text { Copulation duration [min:sec] } \\
\text { Time between mounting and adopting }\end{array} \quad 34: 55 \pm 7: 08(\mathrm{SD})$ & $39: 55 \pm 10: 56(\mathrm{SD})$ & $\mathrm{F}=2.350 ; \mathrm{p}>0.0900$ \\
$\quad$ a stable mating position [sec] & $39 \pm 9(\mathrm{SD})$ & $48 \pm 20(\mathrm{SD})$ & $\mathrm{F}=4.3177 ; \mathrm{p}<0.0035$ \\
$\begin{array}{l}\text { Number of movies suitable for mating angle estimates } \\
\text { Mating angle at 5-10 min after copulation starts }\left[^{\circ}\right]\end{array}$ & $8.55 \pm 1.79(\mathrm{SD})$ & $5.77 \pm 5.33(\mathrm{SD})$ & $\mathrm{F}=8.045 ; \mathrm{p}<0.001$ \\
\hline
\end{tabular}


were recorded at a 100 -fold magnification using a Zeiss Axio Imager Z1 light microscope. External lobe lengths were captured manually and measured using the TpsDIG2.16 program.

\section{Results}

The D. pachea male epandrium is asymmetric

Lobes are part of the D. pachea male epandrium and grow out laterally to the clasper towards the ventral side (Fig. 1a). The base of the lobes merges with the epandrium / hypandrium connection and with a lateral spine. We measured lobe length as the distance between the base of this spine and the distal tip of the lobe and found that lobe length ratio is remarkably reproducible among males of stocks 15090-1698.01 and 15090-1698.04 $(1.44 \pm 0.07$ (SD, n=20) (Fig. 1c and Fig. 2a)).

\section{The D. pachea copulation posture is asymmetric}

In order to quantify mating postures, we filmed mating couples from above and measured the angle between the midlines of male and female during copulation (Fig. 3a). We found that D. pachea couples adopted a characteristic one-sided mating posture. First, males jumped on the female abdomen and moved for $39 \pm 9$ $\sec (\mathrm{SD}, \mathrm{n}=19)$ on the female until encountering a stable mating position. After that, males were observed in a stereotyped angle on the right side of the female. The angle variation was lowest and most reproducible during the time interval of five to ten minutes after the beginning of copulation and was measured to be $8.55^{\circ}$ $\pm 1.79^{\circ}$ (SD, $\mathrm{n}=12$ ) (Fig. 3a). As copulation proceeded, the angle diminished progressively and variation in mating postures increased. The body of the male was oriented towards the right side of the female during the entire copulation. The average copulation duration in our assays was 34:55 \pm 7:08 (min:sec, $n=19)$ (Table 1), which is in agreement with previous studies (Pitnick and Markow, 1994).

One D. pachea stock contains mutant males with symmetric lobes

We found that approximately $20 \%$ of males of the 15090-1698.02 stock possessed symmetric external lobes, with the left lobe being shorter than in wild-type (Fig. 1b-c). In wild-type males, bristles are located throughout the distal parts of both lobes (Fig. 1a) while they are all combined in a single patch in males with symmetric lobes (Fig. 1b). In addition, symmetric mutant lobes appear thicker than normal (Fig. 1b) and symmetric mutant males display a higher range of lobe lengths than wild-type males (Fig. 2).

The copulation position of males with symmetric genitalia is atypical and unsteady

We examined copulation of wild-type and symmetric mutant individuals and dissected their male genitalia afterwards. We considered individuals from the 15090-1698.02 stock as mutants if they fell out of the range of lobe lengths observed in stocks 15090-1698.01 and 15090-1698.04 (Fig. 2). We found that 39\% of symmetric mutant males $(9 / 23)$ were unable to form a stable mating complex within 45 minutes of recording. In contrast, all the asymmetric wild-type males (19/19) accomplished a stable mating complex at the first attempt. Males that failed to settle undertook between 1 and 15 mating attempts with a median of 5 attempts. Those males moved their genitalia back and forth and fell off, or were eventually pushed off by the female with its hind legs. These failed attempts lasted in average $50 \pm 43 \mathrm{sec}(\mathrm{SD}, \mathrm{n}=49)$, with a range of $3 \mathrm{sec}$ to 3 $\min$. The symmetric individuals that failed to copulate had significantly smaller lobes than the symmetric mutant males that achieved copulation (t-tests: left lobe, $\mathrm{t}_{8,13}=3.33, \mathrm{p}<0.0036$; right lobe, $\mathrm{t}_{8,13}=4.33, \mathrm{p}<$ 0.0004; F-tests: left lobe, $\mathrm{F}_{12,7}=1.1081$, $\mathrm{p}>0.4648$; right lobe, $F_{12,7}=0.7204, p>0.7054$ ) (Fig. 3c), indicating that a minimal lobe size is required for mating.

Among the other 14 apparently successful copulations, three symmetric males managed to form a stable mating position at the second attempt and eleven accomplished it at the first trial. In the successful copulations, symmetric males jumped on the female abdomen and moved for $48 \pm 20 \mathrm{sec}(\mathrm{SD}, \mathrm{n}=14)$ on the female until encountering a stable mating position. The time required for male stabilization was significantly more variable than in wild-type (F-test, $\mathrm{F}_{18,13}=4.318$, $\mathrm{p}<0.0035)$. Males with symmetric genitalia displayed a right-sided mating position as previously observed with wild-type males but the variation in position angle was significantly different compared to asymmetric males (F-Test, $\mathrm{F}_{11,10}=7.839, \mathrm{p}<0.0022$ ) (Fig. $3 \mathrm{a}-\mathrm{b}$ and Table 1). The recorded position angle at 5-10 min was $8.55^{\circ} \pm 1.79^{\circ}(\mathrm{SD}, \mathrm{n}=12)$ in wild-type and $5.77^{\circ} \pm$ $5.33^{\circ}(\mathrm{SD}, \mathrm{n}=11)$ in symmetric males.

Although more variable between individuals, the mating angle remained relatively constant for a given 
pair of copulating individuals between 5 and $15 \mathrm{~min}$ after copulation started (Fig. 3a). Since lobe length is variable between mutant individuals (Fig. 2a), we tested whether the mating angle correlates with left lobe length, right lobe length, total lobe length or lobe size ratio. We did not find any correlation between mating angle at 5-10 min and any of these four parameters (Fig. 4), suggesting that the mating angle adopted by a particular symmetric mutant male is determined by factors unrelated to lobe length.

\section{Discussion}

Drosophila pachea males mate on the female's right side

We found that $D$. pachea males mating postures are one-sided, with the male shifted towards the female's right side. This coincides with the observed morphological asymmetry of the male external genitalia, where the left external lobe is longer and thinner than the right lobe.

The mating position of $D$. pachea is somewhat different from the one observed in D. melanogaster after ablation of the mechanosensory clasper bristle (Acebes et al., 2003). In D. pachea, the male first mounts the female from the back in the direction of the female, and then positions itself towards the female's right wing. During copulation, the $D$. pachea male always stands between the two female wings whereas the ablated $D$. melanogaster male grasps the female from the side and does not position itself between female wings. Furthermore, D. melanogaster males with an ablated bristle on the right side mate on the female's left side (Acebes et al., 2003) whereas wild-type D. pachea flies have shorter right lobes and mate on the female's right side. This suggests that the one-sided positioning of the male on top of the female uses different cues in these two species.

\section{Lobe function in mating}

We had the fortune to discover mutant males that possess symmetric lobes, which allowed us to test whether lobe size asymmetry is responsible for the characteristic right-sided mating position of $D$. pachea. We observed that $39 \%$ of the symmetric mutant males failed to copulate within 45 min whereas all the wild-type males did copulate within this time period. The males that failed to copulate had significantly smaller left and right lobes than the symmetric mutant males that did mate. This suggests that a minimal lobe size is required for mating. Males with lobes shorter than this
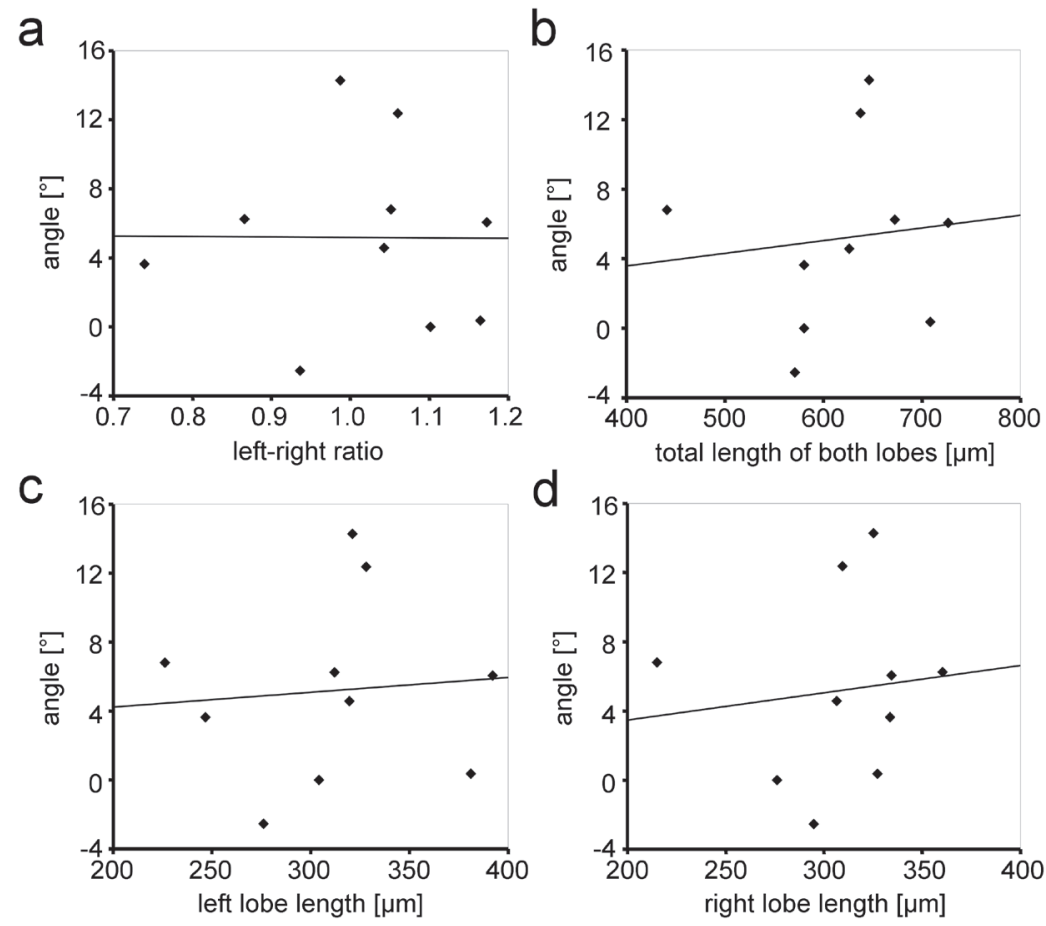

Fig. 4. Absence of correlation between mating angle and lobe length measurements. Scatter plots show the mating angles of symmetric mutant males at 5-10 min after the beginning of copulation, plotted against (a) left-right lobe length ratio (left-right ratio) $(\mathrm{r}=-0.006, \mathrm{p}>$ $0.984)$, (b) total lobe length $(r=0.113, p$ $>0.756)$, (c) left lobe length $(r=0.085, p$ $>0.816)$, and (d) right lobe length $(\mathrm{r}=$ $0.120, p>0.741)$. Each dot corresponds to one individual. 
minimal length might not be able to position themselves correctly on the female or they might not stimulate the female in a correct manner.

We also found that the mating angle in symmetric males that did mate is more variable than in wildtype males. This suggests that lobes are also involved in stabilizing mating complexes. The lobe may grasp or clasp the outer female genitalia, or perhaps stimulate female sensory organs to optimize genitalia coupling. Alternatively, the lobe may act as a proprioceptor and mechanosensory organ that can help males position themselves on the female. Future electron microscopy studies of $D$. pachea male-female mating couples, such as those made in D. melanogaster and its sister species $D$. simulans, $D$. sechellia and $D$. mauritiana (Jagadeeshan and Singh, 2006), may provide interesting information about lobe function during mating.

However, for several reasons we cannot firmly conclude that the mating defects we observed are due to lobe size symmetry. First, the distribution of bristles on the left and right lobe is also altered in symmetric mutant males with respect to wild-type lobes (compare Fig. 1a and 1b). These lobe bristles can have mechanosensory functions that are important for mating complex formation. Second, the mutation(s) responsible for the symmetric lobe mutant phenotype is (are) unknown and may affect other tissues, thus perhaps causing mating behaviour defects. However, we detected no difference in copulation duration between symmetric mutants and wild-type $D$. pachea males (Table 1). Furthermore, symmetric mutant males produced offspring and their viability appeared normal (data not shown). We identified the symmetric mutant phenotype in a 15090-1698.02 stock that had been maintained for two years in our laboratory and found it again in the 15090-1698.02 line reordered from the UC San Diego Drosophila stock center this year. Thus, the mutation has been maintained within the 150901698.02 line for at least two years, suggesting that symmetric mutant males are relatively viable in laboratory conditions. We also point out that the symmetric mutant males cannot represent a contamination with a different species because we obtained normal fertile progeny between these mutant males and wildtype $D$. pachea virgin females. A detailed analysis of the entire body of symmetric mutant flies is needed to make sure that the symmetric mutant phenotype only affects lobes, and that lobe size asymmetry is indeed required to encounter and stabilize an asymmetric posture.
Drosophila pachea males with symmetric genitalia still mate on the female's right side

Intriguingly, we found that symmetric mutant males still mated in a right-sided fashion, although with a higher variation in positions. A few mutant individuals possessed a right lobe slightly longer than the left lobe (Fig. 2a) and these also mated on the female's right side (Fig. 3b). These observations indicate that factors other than lobe size asymmetry must be responsible for the right-sided copulation position of D. pachea.

The male and female internal reproductive organs of D. pachea were described by Patterson (1943) and no left-right asymmetry was reported for those structures. Furthermore, no external structures of the male genitalia other than the lobes have been found to be asymmetric in D. pachea (Pitnick and Heed, 1994). It is possible that a still undescribed asymmetry in female genitalia imposes a right-sided mating position even with symmetric mutants. Alternatively, the onesided mating position may be "hard-wired" as an innate effect of the nervous system of $D$. pachea males or females, even in absence of any organ size asymmetry. Another possibility is that such innate behaviour has evolved after the formation of other asymmetric genital structures that are no longer apparent.

\section{Evolution of mating positions}

In summary, $D$. pachea males mate on the female's right side regardless of their lobe asymmetry or symmetry and mutant males with symmetric lobes have difficulties in forming mating complexes with females. These observations suggest that the asymmetric lobes of $D$. pachea appeared during evolution as an adaptation to optimize asymmetric genitalia coupling during mating. The peculiar right-sided mating position of $D$. pachea may thus have evolved prior to lobe asymmetry. This idea is in perfect agreement with Huber (2010), who proposed one scenario for the evolution of genital asymmetry, in which morphological evolution is the result of asymmetric contacts of male and female genitalia due to changes in mating position in the first place. Whether the right-sided mating position is specific to $D$. pachea or present as well in its closely related species is unknown. Two other species of the D. nannoptera group also have asymmetric male genitalia structures. It would be interesting to examine their mating position and test whether adoption of a right-sided mating position preceded the evolution of species-specific asymmetric genitalia structures. 


\section{Acknowledgements}

We thank Amir Yassin, Therese Markow and Maxi Polihronakis Richmond for attentive discussions, Ilona Grunwald-Kadow for showing us climate controlled chambers, and Nicolas Gompel and his colleagues for introduction to fly courtship and copulation monitoring. We are grateful to Claus Lang for further development and construction of our climate controlled chamber. We also thank the reviewers for their useful comments and suggestions. This work was supported by a CNRS ATIP-AVENIR grant given to VO and by a post-doctoral fellowship from the French Ministry of Foreign and European Affairs to ML.

\section{References}

Acebes A, Cobb M, Ferveur J-F. 2003. Species-specific effects of single sensillum ablation on mating position in Drosophila. Journal of Experimental Biology 206: 3095-3100.

Alexander RD. 1964. The evolution of mating behavior in arthropods. Symposia of the Royal Entomological Society of London 2: 78-94.

Brühl L. 1897. Anatomie und Entwicklungsgeschichte der Geschlechtsausführwege sammt Annexen von Calliphora erythrocephala. Zoologische Jahrbücher, Abteilung für Anatomie und Ontogenie der Thiere 10: 511-618.

Feuerborn HJ. 1922. Das Hypopygium "inversum" und "circumversum” der Dipteren. Zoologischer Anzeiger 55: 89-213.

Heed WB, Kircher HW. 1965. Unique sterol in the ecology and nutrition of Drosophila pachea. Science 149: 758-761.

Huber BA. 2010. Mating positions and the evolution of asymmetric insect genitalia. Genetica 138: 19-25.

Huber BA, Sinclair BJ, Schmitt M. 2007. The evolution of asymmetric genitalia in spiders and insects. Biological Reviews 82: 647-698.

Jagadeeshan S, Singh R. 2006. A time-sequence functional analysis of mating behaviour and genital coupling in Drosophila: role of cryptic female choice and male sexdrive in the evolution of male genitalia. Journal of Evolutionary Biology 19: 1058-70.

McAlpine JF. 1981. Morphology and terminology - adults. Pp. 45-63 in: McAlpline JF, Peterson BV, Shewell GE, Teskey HJ, Vockeroth JR, Wood DM, eds, Manual of Nearctic Diptera, vol. 1. Research branch, agriculture Canada mono- graph No. 27. Ottawa: Canadian Government Publishing Centre.

Meigen JW. 1830. Drosophila. Pp. 81-88 in: Systematische Beschreibung der bekannten europäischen zweiflügeligen Insekten, vol. 6. Hamm: Schulzische Buchhandlung.

Patterson J. 1943. Studies in the genetics of Drosophila III. The Drosophilidae of the southwest. University of Texas Publications 4313: 7-216.

Patterson J, Wheeler M. 1942. Description of new species of the subgenera Hirtodrosophila and Drosophila. University of Texas Publications 4213: 67-109.

Pitnick S, Heed W. 1994. New species of cactus-breeding Drosophila (Diptera: Drosophilidae) in the Nannoptera species group. Annals of the Entomological Society of America 87: 307-310.

Pitnick S, Markow T. 1994. Male gametic strategies: Sperm size, testes size, and the allocation of ejaculate among successive mates by the sperm-limited fly Drosophila pachea and its relatives. The American Naturalist 143: 785-819.

Pitnick S, Markow T, Riedy M. 1991. Transfer of ejaculate and incorporation of male-derived substances by females in the nannoptera species group (Diptera: Drosophilidae). Evolution 45: 774-780.

Schräder T. 1927. Das Hypopygium "Circumversum” von Calliphora erythrocephala. Ein Beitrag zur Kenntnis des Kopulationsapparats der Dipteren. Zoomorphology 8: 1-44.

Suzanne M, Petzoldt AG, Spéder P, Coutelis J-B, Steller H, Noselli S. 2010. Coupling of apoptosis and 1/r patterning controls stepwise organ looping. Current Biology 20: 1773-1778.

Vilela C, Baechli G. 1990. Taxonomic studies on Neotropical species of seven genera of Drosophilidae (Diptera). Mitteilungen der Schweizerischen Entomologischen Gesellschaft 63: 1-332.

Warren JT, Wismar J, Subrahmanyam B, Gilbert LI. 2001. Woc (without children) gene control of ecdysone biosynthesis in Drosophila melanogaster. Molecular and Cellular Endocrinology 181: 1-14.

Wheeler M. 1949. Taxonomic studies on the Drosophilidae. University of Texas Publications 4920: 157-195.

Received: 28 October 2011

Revised and accepted: 22 February 2012

Published online: 31 May 2012

Editor: M. Schilthuizen 